\title{
DOCUMENTS
}

Horst Bartel

\section{DER INTERNE JUNI-ENTWURF ZUM ERFURTER PROGRAMM}

Bisher war es nicht möglich, lückenlos die Genesis des Erfurter Programms zu verfolgen. Nunmehr kann ein für die Entstehung des Erfurter Programms wichtiges Dokument veröffentlicht werden: Der interne Entwurf des Parteivorstandes vom 18. Juni $1891^{1}$ ist jene bisher unbekannte Vorlage, auf die sich die bekannte Kritik Friedrich Engels' aus dem Jahre 1891 bezieht.

Die Ausarbeitung eines neuen Parteiprogramms nach dem Sturz des Sozialistengesetzes war ein Bedürfnis der Partei. Das Gothaer Programm entsprach bereits zur Zeit seiner Annahme in seiner Gresamtkonzeption weder dem theoretisch-ideologischen noch dem praktischpolitischen Entwicklungsstand der sozialistischen Arbeiterbewegung in Deutschland, obwohl es wichtige Forderungen der sozialistischen Bewegung enthielt. ${ }^{2}$ Die „Randglossen zum Programm der deutschen Arbeiterpartei" von Karl Marx und die kritischen Briefe von Friedrich Engels $^{3}$ weisen diese Tatsache eingehend nach. Wenn die deutsche Sozialdemokratie zu einer marxistischen Massenpartei wurde, so vollzog sich diese Entwicklung trotz des Gothaer Programms und gegen seine ideologisch-politische Gesamtkonzeption. Die Kritik am Gothaer Programm wurde eigentlich bereits auf dem Parteitag von 1875

1 Im folgenden interner Juni-Entwurf genannt. NachlaB Karl Kautsky, K A 21, Internationaal Instituut voor Sociale Geschiedenis, Amsterdam. Herrn Direktor Prof. Dr. de Jong danke ich für die Genehmigung zur Veröffentlichung des Dokuments.

2 Vgl. dazu ausführlicher Geschichte der deutschen Arbeiterbewegung in acht Bänden, Bd. 1, Berlin 1966, S. 334ff; Erich Kundel, Marx und Engels im Kampf um die revolutionäre Arbeitereinheit. Zur Geschichte des Gothaer Vereinigungskongresses von 1875, Berlin 1962; Ernst Engelberg, Deutschland von 1871 bis 1897. Deutschland in der Úbergangsperiode zum Imperialismus, Berlin 1965 , S. $138 \mathrm{ff}$.

${ }^{3}$ Engels an Bebel, 18./28.3.1875, in: Karl Marx und Friedrich Engels, Werke, Dietz Verlag, Berlin 1966, Bd. 34, S. 125ff. Engels an Bracke, 11.10.1875, in: Ebenda, S. $155 \mathrm{ff}$. 
eröffnet und riß seitdem nicht ab. ${ }^{1}$ Bernstein selbst sprach 1875 von „Konzessionen"2 und schrieb 1891: „Hat nicht das Gothaer Programm Punkte, die nur deshalb nicht geschadet haben, weil wir von Anfang an konsequent im entgegengesetzten Sinne handelten?"3 Die Geschichte der deutschen Sozialdemokratie von 1875 bis 1891 ist daher auch eine Geschichte der Überwindung des Gothaer Programms.

Die Delegierten des Parteitages von St. Gallen 1887 beschlossen Ausdruck des innerparteilichen Klärungsprozesses ${ }^{4}$ - die Ausarbeitung eines neuen Programms. Sie wählten drei ehemalige Eisenacher, Ignaz Auer, August Bebel und Wilhelm Liebknecht, zu Mitgliedern der Redaktionskommission. ${ }^{5}$ Die Kommission konnte auf dem Parteitag von Halle 1890 noch kein Programm vorlegen. Wilhelm Liebknecht erstattete vor dem Parteitag einen Bericht über die Programmfrage, in dem er - teils gestützt auf die noch unveröffentlichte Marxsche Kritik des Gothaer Programms, teils gegen sie polemisierend - verschiedene Gesichtspunkte für das neu auszuarbeitende Programm darlegte. Liebknecht hob als Grundforderung für das zukünftige Programm hervor: .... . wissenschaftliche Präzision, welche ein Programm unserer Partei unbedingt haben sollte - das Programm einer Partei, die sich mit Recht als die Partei des wissenschaftlichen Sozialismus bezeichnet."

1 Vgl. dazu Wolfgang Schröder und Gustav Seeber, Zur Vorbereitung des Erfurter Programms, in: ZfG, XIV. Jg. 1966, H. 7, S. 1117ff.

2 Vgl. August Bebel, Aus meinem Leben, Bd. 2, Berlin 1953, S. 276.

Eduard Bernstein an Karl Kautsky, 19.2.1891, in: Nachlaß Karl Kautsky, IISG Amsterdam. Im Gegensatz dazu stellte Bernstein wenige Jahre später unter Berufung auf Kautsky das Gothaer Programm als Ausdruck des Reifegrades der politischen Arbeiterbewegung Deutschlands hin. Vgl. Eduard Bernstein, Zur Vorgeschichte des Gothaer Programms, in: Die Neue Zeit, 15. Jg. (1896-1897), 1. Bd., S. 466ff; Eduard Bernstein, Sozialdemokratische Lehrjahre, Berlin 1928, S. 46. Diese These wurde aufgegriffen u.a. von Willi Eichler, Einleitung zu: Programme der deutschen Sozialdemokratie, Bonn 1963, S. 11, und Susanne Miller, Das Problem der Freiheit im Sozialismus. Freiheit, Staat und Revolution in der Programmatik der Sozialdemokratie von Lassalle bis zum Revisionismusstreit, Frankfurt/M. 1964, S. 79.

4 Vgl. dazu Ernst Engelberg, Revolutionäre Politik und Rote Feldpost 18781890, Berlin 1959; Heinrich Gemkow, Friedrich Engels' Hilfe beim Sieg der deutschen Sozialdemokratie über das Sozialistengesetz, Berlin 1957, und Horst Bartel, Marx und Engels im Kampf um ein revolutionäres deutsches Parteiorgan 1879 bis 1890 , Berlin 1961.

- Der Beschlu $B$ des Parteitages neuerdings abgedruckt bei Schröder und Seeber, a.a.O., S. 1128-1129. Vgl. zu dem folgenden auch mein Nachwort zu Karl Kautsky, Das Erfurter Programm, Berlin 1965, S. $261 \mathrm{ff}$.

- Protokoll über die Verhandlungen des Parteitages der Sozialdemokratischen Partei Deutschlands, abgehalten zu Halle a.S. vom 12. bis 18. Oktober 1890, Berlin 1890, S. 160 . 
Parteitag beschloB, daß der Parteivorstand ein Programm auszuarbeiten und mindestens drei Monate vor dem nächsten Parteitag der Mitgliedschaft zu unterbreiten habe. ${ }^{1}$ „Das Programm”, so sagte Liebknecht, „muß die Kollektivarbeit sämtlicher Parteigenossen sein."2

Entscheidend griff jetzt Friedrich Engels in die Proprammdiskussion ein. In der zweiten Januarhälfte 1891 veröffentlichte er in der Neven Zeit unter dem Titel ,Zur Kritik des sozialdemokratischen Parteiprogramms aus dem Nachlaß von Karl Marx" die Marxschen Randglossen zum Gothaer Programmentwurf. ${ }^{3}$ So unterschiedlich die Aufnahme dieser Veröffentlichung durch die führenden Sozialdemokraten war, ${ }^{\mathbf{4}}$ die tatsächliche Wirkung war eindeutig. Mit vollem Recht konnte Friedrich Engels erklären: „Es macht jede Halbheit und Phrasenhaftigkeit im nächsten Programm unmöglich und liefert unwiderstehliche Argumente, die die Meisten von ihnen [Vorstandsmitglieder - H.B.] vielleicht kaum den Mut gehabt hätten aus eigener Initiative vorzubringen."5 Zur Unterstützung der Programmdiskussion veröffentlichte Friedrich Engels im Frühjahr 1891 die meisterhafte Darstellung der Geschichte und der Lehren der Pariser Kommune von Karl Marx „Der Bürgerkrieg in Frankreich”. Das von Engels hinzugefügte Vorwort wurde in der Neuen Zeit und auch im Vorwärts abgedruckt. ${ }^{6}$

Im Mai 1891 arbeitete Wilhelm Liebknecht den Entwurf des neuen Programms aus. ${ }^{7}$ Dazu formulierte Bebel einen Gegenentwurf, den Liebknecht wiederum für eine neue Fassung benutzte. Daraus entstand schließlich durch die kollektive Arbeit des Vorstandes ein Programmentwurf, der am 18. Juni 1891 an die Fraktionsmitglieder und an hervorragende Parteiführer und Theoretiker versandt wurde. ${ }^{8}$ Dieser am 18. Juni 1891 vom Parteivorstand mit dem Vermerk

1 Ebenda, S. 181.

2 Ebenda, S. 159.

${ }^{3}$ Die Neue Zeit, 9. Jg. (1890-1891), 1. Bd., S. 561ff.

4 Hier soll nicht ausführlich auf diese Frage eingegangen werden. Vgl. dazu Friedrich Engels' Briefwechsel mit Karl Kautsky, hrsg. von Benedikt Kautsky, Wien 1955, S. 268ff., und August Bebels Briefwechsel mit Friedrich Engels, hrsg. von Werner Blumenberg, London-The Hague-Paris 1965, S. 406ff.

5 Engels an Kautsky, 3.2.1891, in: Friedrich Engels' Briefwechsel mit Karl Kautsky, S. 272.

Die Neue Zeit, 9. Jg. (1890-1891), 2. Bd., S. 33ff., und Vorwärts, Nr. 27 v. 1.2.1891 und Nr. 28 v. 3.2.1891.

7 Kautsky an Engels, 4.6.1891, in: Friedrich Engels' Briefwechsel mit Karl Kautsky, S. 299.

${ }^{8}$ So berichtet Bebel an Engels, 18.6.1891, in: August Bebels Briefwechsel mit Friedrich Engels, S. 420-421. Die von Bebel an Engels gleichzeitig gesandten ersten Entwürfe von Liebknecht und Bebel sind bisher nicht aufgefunden worden. 
„streng vertraulich" verschickte Entwurf, der als Manuskriptdruck versandt wurde, ist in dem uns vorliegenden Exemplar mit Randbemerkungen Kautskys versehen, die in Anmerkungen wiedergegeben werden.

Auch an Friedrich Engels wurde ein solcher Entwurf versandt, den er nicht nur ausführlich kritisierte, sondern auch bearbeitete. ${ }^{1}$ Engels stellte fest, daß sich diese Vorlage sehr vorteilhaft von dem bisherigen Programm unterscheide. ${ }^{2}$ Spezifisch lassallesche und vulgärsozialistische Auffassungen seien faktisch beseitigt. Der Entwurf stehe, so bemerkte Engels in der Einleitung zu seiner Kritik, „nach seiner theoretischen Seite im ganzen auf dem Boden der heutigen Wissenschaft und läßt sich von diesem Boden aus diskutieren."3 Damit gewann die Diskussion des Entwurfs auch innerhalb der marxistischen Führungskräfte der Partei einen ganz anderen Charakter als etwa 1875.4 Sowohl zwischen Liebknecht und Bebel - die ja die Hauptarbeit geleistet hatten - als auch zwischen den Mitgliedern des Parteivorstandes und Friedrich Engels bestand von vornherein Übereinstimmung über die Prinzipien und damit über den Inhalt des Programms.

$\mathrm{Zu}$ dem vom Parteivorstand versandten Entwurf gingen nun von verschiedenen Seiten Änderungs- bzw. Ergänzungsvorschläge ein. Die wichtigste kritische Stellungnahme schrieb ohne Zweifel Friedrich Engels. ${ }^{5}$ Vergleicht man nun den hier abgedruckten Entwurf, die Kritik von Engels und den im Vorwärts am 4. Juli $1891^{6}$ veröffent-

1 Engels an Kautsky, 29.6.1891, in: Friedrich Engels' Briefwechsel mit Karl Kautsky, S. 304. In diesem Zusammenhang sei auf einige Irrtümer hingewiesen, die sich bis in die neueste Literatur eingeschlichen haben. Die Arbeit von Friedrich Engels, die unter dem Titel „Zur Kritik des sozialdemokratischen Programmentwurfs 1891" bekannt geworden ist (Karl Marx und Friedrich Engels, Werke, Bd. 22, Berlin 1963, S. $227 \mathrm{ff}$.), bezieht sich auf den am 18. Juni als "Streng vertraulich" versandten Entwurf. Werner Blumenberg verweist irrtümlich auf den am 4. Juli verötfentlichten Entwurf (August Bebels Briefwechsel mit Friedrich Engels, S. 421, Anm. 1), und Wolfgang Abendroth bezieht die Kritik auf den Entwurf Kautskys (Wolfgang Abendroth, Aufstieg und Krise der deutschen Sozialdemokratie, Frankfurt/M. 1964, S. 81, Anm. 51). 2 Werke, Bd. 22, S. 227.

3 Ebenda.

4 VI. Geschichte der deutschen Arbeiterbewegung, Bd. 1, a.a.O., und Erich Kundel, a.a.O.

${ }^{5}$ Kautsky hatte einen Gegenentwurf an den Vorstand geschickt, der aber wegen seiner Länge nicht akzeptiert wurde (Bebel an Engels, 12. Juli 1891, a.a.O., S. 423). Dieser Entwurf ist nicht identisch mit dem schließlich in Erfurt vorgelegten Entwurf Kautskys (Vgl. Engels an Kautsky, 28. Sept. 1891, a.a.O., S. 307).

- Vorwärts, Nr. 153 v. 4.7.1891. 
lichten Entwurf des Parteivorstandes, so kann man die Bemerkung Bebels bestätigen, daß der erste Teil des Entwurfs vom 4. Juli faktisch ein Entwurf Engels' war. ${ }^{1}$

Auf Grund der Kritik von Engels erfuhr nun der am 18. Juni versandte Vorstandsentwurf eine Straffung. Darüber hinaus erreichte Engels in einigen wesentlichen Punkten eine klarere Fassung im Begrifflichen, was - eben im Hinblick darauf, daß der Marxismus eine geschlossene Wissenschaft ist und daher jeder Begriff auch einen bestimmten Tatbestand, eine gesellschaftliche Realität oder ein gesellschaftliches Verhältnis ausdrückt - wichtig war. Im ganzen zeigt ein Vergleich, daß doch eine wesentliche Übereinstimmung zwischen den im internen Juni-Entwurf und den von Engels vertretenen Auffassungen über die kapitalistische Gesellschaft und die Rolle des Proletariats und den Aufgaben der Partei bestand.

Hauptpunkt der Kritik Engels' am internen Juni-Entwurf war das Verhältnis von Demokratie und Sozialismus. Bei diesem für die Strategie und Taktik der Partei wichtigen Problem ging es um das demokratische Etappenziel des sozialistischen Kampfes, die demokratische Republik. Wenn Engels schrieb, „da $\beta$ unsre Partei und die Arbeiterklasse nur zur Herrschaft kommen kann unter der Form der demokratischen Republik,"2 so faßte er seine in den achtziger Jahren gegenüber Bernstein und Bebel ausführlich dargelegten Gedanken zusammen. Ihm kam es dabei auf folgenden entscheidenden Gesichtspunkt an: „Die bürgerliche Republik ... dient uns zunächst zur Eroberung der großen Massen der Arbeiter für den revolutionären Sozialismus. .."3 Diese Problematik war in dem Entwurf - es betrifft vornehmlich die Punkte 1-3 der politischen Forderungen - nicht klar ausgeführt. Angesichts der politischen Zustände in Deutschland schlug Engels vor, die Forderung nach der demokratischen Republik mit dem Satz zu umschreiben: „Konzentration aller politischen Macht

1 Vgl. die Rede Bebels am 16. Juli 1891, abgedruckt in Schröder und Seeber, Zur Vorbereitung des Erfurter Programms, a.a.O., S. 1136. Als Entwurf Engels' bezeichnete Bebel ohne Zweifel die in der „Beilage zu Abschnitt I” (Werke, Bd. 22, S. 239-240) vorgenommene Zusammenfassung. Dazu schrieb Engels in seinen ausführlicheren Bemerkungen (ebenda, S. 228): „Ich will versuchen, diesen ganzen Passus etwas kürzer zu fassen, und, wenn es mir gelingt, ihn beilegen oder nachschicken. .." An Victor Adler berichtete Bebel, daß der Entwurf von Engels ,sich an den unseren anlehnte u. mehrere Absätze ganz acceptierte ..." (Bebel an Adler, 7.7.1891, in: Victor Adler, Briefwechsel mit August Bebel und Karl Kautsky, Wien 1954, S. 74).

${ }^{2}$ Werke, Bd. 22, S. 235.

${ }^{3}$ Engels an Bernstein, 27.8.1883, in: Eduard Bernstein, Die Briefe von Friedrich Engels an Eduard Bernstein, Berlin 1925, S. 129-130. Vgl. auch August Bebels Briefwechsel mit Friedrich Engels, S. $193 \mathrm{ff}$. 
in den Händen der Volksvertretung". 1 Sein Vorschlag wurde in dieser Form nicht akzeptiert, ${ }^{2}$ aber unter Punkt 2 wurde hinzugefügt: „Selbstverwaltung des Volks im Reich, Staat, Provinz und Gemeinde. Jährliche Steuerbewilligung, Recht der Steuerverweigerung."3 Damit kam das Programm den Vorstellungen von Engels sehr nahe.

Es war keinesfalls so, daß etwa die führenden Vertreter der Sozialdemokratie der Frage der Republik bzw. dem Problem der Etappen im Kampf um die Macht keine Aufmerksamkeit schenkten. Kautsky hat $\mathrm{zu}$ den Forderungen 2 und 3 des Entwurfs am 18. Juni an den Rand notiert: „Republik”. ${ }^{4}$ Bernstein machte auch auf das Problem aufmerksam - sicherlich in Anlehnung an die Ausführungen von Engels -, als er wenigstens ,die Verwandlung der Volksvertretung in einen wirklich beschließenden Körper" aufzunehmen empfahl, "wenn man schon nicht die Republik fordert". ${ }^{5}$ Und Bebel schrieb an Engels: „Daß die Forderungen des Programms nicht mit dem Königtum verwirklicht werden, darüber täuscht sich niemand."6 Wilhelm Liebknecht faßte diese Ansichten schließlich zusammen, wenn er den grundsätzlich revolutionären Charakter des zweiten Teils des Programms und damit seinen Zusammenhang mit den im ersten Teil dargelegten Zielen der Sozialdemokratie umriß: „Wir haben hier zum ersten Male ausgesprochen, da $B$ wir nur eine solche Staats- und Regierungsform, die auf der breitesten demokratischen Basis beruht, direkt aus dem Volke hervorgegangen ist und von dem Volke kontrolliert wird, mit dem Prinzip der Volkssouveränität in Harmonie stehend erachten. ... wir verlangen, daß alle Einrichtungen und Gesetze, welche den Äußerungen und Betätigungen des Volkswillens jetzt entgegenstehen, beseitigt werden. $\mathrm{Daß}$ hiermit eine vollständige Umgestaltung unserer

1 Werke, Bd. 22, S. 235.

$2 \mathrm{DaB}$ die von Engels vorgeschlagene Formulierung nicht aufgenommen wurde, verwundert etwas, zumal in dem programmatischen Wahlaufruf von 1887 „für die Volksvertretung die äußersten Machtbefugnisse” gefordert worden waren. Vgl. den Wahlaufruf in: Die Sozialdemokratie im Deutschen Reichstag. Tätigkeitsberichte und Wahlaufrufe aus den Jahren 1871 bis 1893, Berlin 1909, Reproduktion Berlin 1966, S. 250.

3 Entwurf des Parteivorstandes (4. Juli 1891), in: Protokoll über die Verhandlungen des Parteitages der Sozialdemokratischen Partei Deutschlands. Abgehalten zu Erfurt vom 14. bis 20. Oktober 1891, Berlin 1891, S. 15.

Siehe S. 301, Textnote a. Die Randbemerkung Kautskys geht offensichtlich nicht auf Engels zurück, da Kautsky die Programmkritik von Engels erst durch einen Brief von Bernstein vom 6.8.1891 erhielt. Bernstein an Kautsky, 6.8.1891, in: NachlaB Karl Kautsky, IISG.

${ }^{5}$ Bernstein an Kautsky, 26.6.1891, in: Ebenda.

- Bebel an Engels, 12.7.1891, in: August Bebels Briefwechsel mit Friedrich Engels, S. 424. 
jetzigen Staatseinrichtungen verbunden ist, begreift Jeder auf den ersten Blick. Es bedeutet die absolute Demokratisierung der politischen Einrichtungen in Deutschland."1

Allerdings wurde die volle Bedeutung der Forderung nach der demokratischen Republik als Etappenziel im Kampf um die sozialistische Gesellschaftsordnung selbst von den marxistischen Führern der deutschen Sozialdemokratie nicht erkannt. ${ }^{2}$ Immerhin erreichte aber Friedrich Engels mit seiner Kritik, daß diese Frage Gegenstand der Diskussion wurde und größere Klarheit erreicht werden konnte. So konnte der sozialdemokratische Parteivorstand am 4. Juli 1891 einen Entwurf veröffentlichen, dessen marxistische Prinzipien den Charakter der Programmdiskussion in der Partei und der Arbeiterklasse Deutschlands bestimmten.

Der nachfolgend erstmals veröffentlichte interne Juni-Entwurf des Erfurter Programms ermöglicht nun, die Kritik Friedrich Engels' am sozialdemokratischen Programmentwurf in vollem Umfang zu verstehen. Ferner beweist das Dokument, da $B$ Engels in wesentlich größerem Maße, als bisher angenommen werden konnte, direkten Einfluß auf die endgültige Fassung des Erfurter Programms nahm. Es demonstriert somit die dominierende Rolle, die der Marxismus bei der Ausarbeitung der programmatischen Grundsätze der sozialistischen Arbeiterbewegung Deutschlands ausübte.

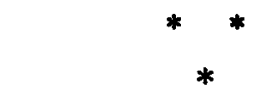

\section{Streng vertraulich!}

W.G.! Wir senden Ihnen anbei den Entwurf unseres dem nächsten Parteitag vorzulegenden Parteiprogramms mit der Bitte um gefl. Aeußerung Ihrer Meinung unter Beifügung etwaiger Abänderungsvorschläge. Wir bitten aber, diesen Entwurf als streng vertraulich $\mathrm{zu}$ behandeln, da derselbe erst nach Berücksichtigung der etwa sich ergebenden Bedenken und Einwände zur weiteren Kenntniß der Genossen und damit der Oeffentlichkeit überhaupt gelangen soll. Ihren Rückäußerungen sehen wir bis spätestens

\section{Sonnabend, den 27. Juni}

entgegen, weil - laut Haller Kongreß-Beschluß - der Programm-Ent-

1 Protokoll Erfurt, S. 346.

${ }^{2}$ Eine speziellere Untersuchung zu dieser Problematik wird der Verfasser demnächst vorlegen. 
wurf mindestens 3 Monate vor dem nächsten Parteitag der öffentlichen Diskussion unterbreitet werden soll.

Mit sozialdemokratischem Gruß

Der Partei-Vorstand

\section{ENTWURF EINES PROGRAMMS \\ DER}

SOZIALDEMOKRATISCHEN PARTEI DEUTSCHLANDS.

Die Trennung des Arbeiters von den Arbeitsmitteln - Grund und Boden, Bergwerke, a Gruben, Minen, Maschinen und Werkzeuge, Verkehrsmittel - und deren Uebergang in den Alleinbesitz eines Theils der Gesellschaftsglieder hat zur Spaltung der Gesellschaft in zwei Klassen $:^{\mathbf{b}}$ die arbeitende und die besitzende geführt.

In den Händen der Alleinbesitzer (Monopolisten) sind die für den Bestand der Gesellschaft nothwendigen Arbeitsmittel zu Mitteln der Ausbeutung geworden. Die hierdurch bedingte Abhängigkeit der Arbeiter von den Alleinbesitzern der Arbeitsmittel ist die Grundlage der Knechtschaft in jeder Gestalt: der politischen Rechtlosigkeit, des sozialen Elends, der physischen Verkümmerung, der geistigen Herabwürdigung.

Unter der Herrschaft der Alleinbesitzer - Kapitalisten, Großgrundbesitzer, Bourgeois - nimmt die Anhäufung der Arbeitsmittel und des durch die Ausgebeuteten erzeugten Reichthums in den Händen der Kapitalisten mit wachsender Geschwindigkeit $\mathbf{z u}$ - immer ungleicher wird die Vertheilung des Arbeitsprodukts, immer größer die Zahl und das Elend der Proletarier, immer massenhafter die Armee der überschüssigen Arbeiter, immer schroffer der Klassengegensatz, immer erbitterter der Klassenkampf, welcher die moderne Gesellschaft in zwei feindliche Heerlager trennt und das gemeinsame Merkmal aller Industrieländer ist.c

Die im Wesen der kapitalistischen Privatproduktion begründete Planlosigkeit erzeugt jene immer länger andauernden Krisen und Arbeitsstockungen, welche die Lage der Arbeiter noch verschlimmern, den Ruin weiter Volksschichten nach sich ziehen, allgemeine wirthschaftliche und politische Unsicherheit zur Folge haben und die ganze Gesellschaft in Unruhe und Verwirrung stürzen.

Diesem Zustand, der von Tag zu Tag unerträglicher wird, durch

Randnotiz Kautskys Rohstoffe.

b Bauern? Handwerker.

c Am Rand des gesamten Abschnittes technische Umwälzungen 
Beseitigung der Ursachen ein Ende zu machen und die Befreiung der Arbeiterklasse zu erringen, ist das Ziel und die Aufgabe der Sozialdemokratie.

Die Sozialdemokratische Partei Deutschlands erstrebt demgemä $B$ die Umwandlung der Arbeitsmittel - Grund und Boden, Bergwerke, Minen, Gruben, Maschinen und Werkzeuge, Verkehrsmittel - in Gemeineigenthum der Gesellschaft und die Umwandlung der kapitalistischen Privatproduktion in sozialistische Gemeinproduktion, für welche die kapitalistische Gesellschaft selbst die materiellen und geistigen Bedingungen schafft.

Die Befreiung der Arbeiterklasse kann nur das Werk der Arbeiterklasse selbst sein, weil alle übrigen Klassen und Parteien auf dem Boden des Kapitalismus stehen und trotz der Interessenstreitigkeiten unter sich doch die Erhaltung und Stärkung des Kapitalismus zum gemeinsamen Ziel haben. ${ }^{a}$

In der Erkenntni $B$, da $B$ die Befreiung der Arbeiterklasse nicht eine nationale, sondern eine soziale $\mathbf{b}^{\mathbf{b}}$ Aufgabe ist, an der die Arbeiter aller Kulturländer gleichmäßig betheiligt sind, und deren Lösung nur durch internationales Zusammenwirken erreicht werden kann, fühlt und erklärt die sozialdemokratische Partei Deutschlands sich eins mit den klassenbewußten Arbeitern aller übrigen Länder.

Die sozialdemokratische Partei hat nichts gemein mit dem sogenannten Staatssozialismus: dem System der fiskalischen Verstaatlichung, das den Staat an die Stelle des Privat-Unternehmers setzt, aber das Doppeljoch ökonomischer Ausbeutung und politischer Unterdrückung auf dem Arbeiter lasten läßt.

Die sozialdemokratische Partei kämpft nicht für neue Klassenprivilegien und Vorrechte, sondern für die Abschaffung der Klassenherrschaft und für das gleiche Recht Aller ohne Unterschied des Geschlechts und der Abstammung. In ihrem Kampf für die Befreiung der Arbeiterklasse, dem Kampf für die Befreiung der Menschheit, verficht die Sozialdemokratie alle Forderungen, Maßregeln und Einrichtungen, welche die Lage des Volkes im Allgemeinen und der Arbeiterklasse im Besonderen zu verbessern geeignet sind.

Die sozialdemokratische Partei Deutschlands tritt deshalb ein für folgende Forderungen:

1. Allgemeines gleiches direktes Wahl- und Stimmrecht mit geheimer Stimmabgabe aller über 21 Jahre alten Staatsangehörigen ohne Unterschied des Geschlechts für alle Wahlen und Abstimmungen. Einführung des Proportionalwahlsystems. Fest-

a Am Rand des gesamten Abschnittes Bauern, Handwerker

b Das Wort soziale von Kautsky gestrichen 
setzung der Wahlen und Abstimmungen auf einen Sonn- oder Feiertag.

2. Direkte Gesetzgebung durch das Volk, das heißt Vorschlagsund Verwerfungsrecht des Volks.

3. Entscheidung über Krieg und Frieden durch die gewählten Vertreter des Volks. Errichtung eines internationalen Schiedsgerichts. ${ }^{\mathrm{a}}$

4. Abschaffung aller Gesetze, welche die freie Meinungsäußerung und das Recht der Vereinigung und Versammlung einschränken oder unterdrücken.

5. Abschaffung aller Zuschüsse aus öffentlichen Mitteln zu kirchlichen und religiösen $Z$ wecken. Die kirchlichen und religiösen Gemeinschaften sind als Privatvereinigungen zu betrachten.

6. Weltlichkeit der Schule. Obligatorischer Besuch der öffentlichen Volksschulen. Unentgeltlichkeit des Unterrichts und der Lehrmittel in allen öffentlichen Bildungsanstalten. ${ }^{\mathbf{b}}$

7. Erziehung zu allgemeiner Wehrhaftigkeit. Volkswehr an Stelle der stehenden Heere.

8. Unentgeltlichkeit der Rechtspflege und der Rechtshülfe. Rechtsprechung durch vom Volk gewählte Richter.c

9. Unentgeltlichkeit der ärztlichen Hülfeleistung und der Arzneimittel.

10. Progressive Einkommen-, Kapital- und Erbschaftssteuer für die Bestreitung aller öffentlichen Ausgaben, soweit diese durch Steuern zu decken sind. Abschaffung aller indirekten Steuern, Zölle und sonstigen wirthschaftspolitischen Maßnahmen, welche die Interessen der Gesammtheit den Interessen einer bevorzugten Minderheit opfern.

Zum Schutze der Arbeiterklasse fordert die Sozialdemokratische Partei Deutschlands:

1. Eine wirksame nationale und internationale Arbeiterschutzgesetzgebung auf folgender Grundlage:

a) Festsetzung eines höchstens acht Stunden betragenden Normalarbeitstags.

b) Verbot der gewerblichen Arbeit für Kinder unter 14 Jahren;

c) Verbot der Nachtarbeit, außer für solche Industriezweige, die ihrer Natur nach, aus technischen Gründen oder aus Gründen der öffentlichen Wohlfahrt Nachtarbeit erheischen;

d) Verbot der Frauenarbeit in Industriezweigen, deren Betriebs-

a Punkt 2 und 3 durch Klammer verbunden und mit dem Wort Republik versehen

b Punkt 5 und 6 durch Strich verbunden und mit sehr gut versehen

c Daneben? Unschuldig Verurteilte u. Verhaftete, Abschaffung der Todesstrafe. 
weise nachtheilig auf den Organismus der Frauen einwirkt;

e) Verbot der Nachtarbeit für Frauen und jugendliche Arbeiter unter 18 Jahren;

f) eine ununterbrochene Ruhepause von mindestens 36 Stunden in jeder Woche für jeden Arbeiter;

g) Verbot des Trucksystems;

h) Ueberwachung aller Werkstätten und industriellen Betriebe, mit Einschluß der Hausindustrie durch vom Reich besoldete Aufsichtsbeamte.

2. Sicherstellung des Koalitionsrechts gegen Uebergriffe der Unternehmer.a

3. Uebernahme der gesammten Arbeiterversicherung durch das Reich, mit maßgebender Mitwirkung der Betheiligten an der Verwaltung. ${ }^{\mathrm{b}}$

Zur Regelung und Ueberwachung der Arbeitsverhältnisse fordert die sozialdemokratische Partei Deutschlands ein Reichs-Arbeitsamt, Bezirks-Arbeitsämter und die Bildung von Arbeitskammern, deren Mitglieder zur einen Hälfte von den Arbeitern, zur andern von den Unternehmern $\mathrm{zu}$ wählen sind.

b Registrierung. 